\title{
LOGISTIC CIRCULATION WITH RISKS IN TRANSPORTATION OF AGRICULTURAL PRODUCTS
}

\author{
Vassil Sgurev, Stanislav Drangajov ${ }^{*}$ and Stefan Koynov \\ Intelligent Systems Department, \\ Institute of Information and Communication Technologies, \\ Bulgarian Academy of Sciences, \\ Acad. Georgi Bonchev St., Bl. 25A, 1113 Sofia, Bulgaria, \\ e-mails:vsgurev@gmail.com; sgrangajov@gmail.com; \\ stefan.koynov@gmail.com
}

\begin{abstract}
This paper is devoted to the problem of optimal transportation of agricultural products through a network of loading, intermediate and unloading points through the circulation of vehicles and taking into account the capacity and the risk of adverse events in different parts of the network. The risk is considered as a product of two measures - the volume of agricultural transport production on a given section of the network and the probability of adverse events on that section. To describe the transport process, the use of circulation in a generalized two-product network flow with bandwidth and risk function is proposed. A method is described by which the minimal number of necessary means of transport for transportation of all production at total minimal transportation costs and for risk reduction insurance can be determined. It is proposed that the whole process for optimization of the transport activity be controlled by a computer system and periodically updated by recalculating the schedule according to the proposed method.

Keywords: two-product network flow, risk function, generalized network flow, maximal circulation with minimal value, closed logistics system.
\end{abstract}

\section{INTRODUCTION}

During the intensive campaigns for harvesting agricultural products from fields and greenhouses and transport to receiving points, a number of problems arise related to the optimal use of the fleet, the minimization of delivery time, as well as the total cost of these operations [1]. Most often the vehicles circulate

* Corresponding author.

DOI: 10.7546/EngSci.LVII.20.04.05

Engineering Sciences, LVII, 2020, No. 4 
between loading, intermediate and unloading points, where it is necessary to draw up optimal for the whole system schedules for the movement of vehicles. The criterion for this is the minimization of the total value of freight transport operations and the costs of insurance to reduce the risks on the network. It is often necessary to minimize the number of vehicles used along their routes.

On the other hand, in the movement of vehicles and loading and unloading operations there are various risks, the cost of which must be taken into account in the optimization of management processes. Similarly, in both natural disasters and man-made events, the risk is usually considered as a product of two measures - the amount of transported product (resource) between the start and end points of a section and the probability of an adverse event when crossing that section $[2,3]$.

In the present work, a circulating generalized two-product network flow with gains and losses has been developed to describe various freight and transport activities in the transportation of agricultural products. The first of the products in this model describes the movement of this production (resource), and the second - the risks that arise from such movement. Both products require "flow", i.e. compliance with the requirement for equality of the quantities of incoming and outgoing product for each peak.

Agricultural production is transported by discrete, usually road transport, which in the two-product model is considered as a continuous flow.

\section{PROBLEM FORMALIZATION AND METHODOLOGY}

For the formal description of the developed model it is necessary to enter the following designations $[4,5]$ :

$G(X, U)$ - a directed graph containing a plurality of vertices $X=\left\{x_{i} / i \in\right.$ $I\}$ and a set of $\operatorname{arcs} U=\left\{x_{i j} /(i, j) \in G\right\}$ in which $I$ is the set of indices of all vertices $X, I=\left\{x_{i} / x_{i} \in I\right\}$ and $G$ is the set of pairs of indices of all arcs $U$ of the graph $G=\left\{(i, j) / x_{i j} \in U\right\}$ where each arc has an initial vertex $x_{i}$ and an end vertex $x_{j}$;

$f_{i j}-$ network flow of the resource on the arc $x_{i j}$;

$r_{i j}-$ arc flow function of the risk on the arc $x_{i j}$ or, in short, the risk on the same arc:

$$
r_{i j}=P_{i j} f_{i j}, \quad 0 \leq P_{i j} \leq 1, \quad(i, j) \in G
$$




$$
\begin{aligned}
P_{i j}- & \text { the probability of occurrence of an adverse event dur- } \\
& \text { ing the transportation of the resource } f_{i j} \text { along the } \\
& \text { } \text { ainbow } r_{i j} ;
\end{aligned}
$$

In order to comply with the constraints by resource $\left\{C_{i j}^{\prime}\right\}$ and by risk $\left\{C_{i j}^{\prime}\right\}$ it is necessary that:

$$
C_{i j}^{s}=\min \left[C_{i j}^{\prime}, C_{i j}^{\prime \prime} / P_{i j}\right], \quad(i, j) \in G,
$$

$C_{i j}^{s}$ - coordinated resource throughput, which respects both throughputs - by resources $C_{i j}^{\prime}$ and by risks $C_{i j}^{\prime \prime}$;

$$
C_{i j}^{r}=\min \left[P_{i j} C_{i j}^{\prime}, C_{i j}^{\prime \prime}\right], \quad(i, j) \in G,
$$

$C_{i j}^{r}$ - coordinated risk throughput, which is saved and two throughputs - by risk $C_{i j}^{\prime \prime}$ and by resource $C_{i j}^{\prime}$.

If the two sides of the first of the above two equations are multiplied by the probability $P_{i j}$, the following equations will be reached:

$$
C_{i j}^{s} P_{i j}=\min \left[P_{i j} C_{i j}^{\prime}, C_{i j}^{\prime \prime}\right]=C_{i j}^{r} \text { or } C_{i j}^{r}=P_{i j} C_{i j}^{s}, C_{i j}^{s}=C_{i j}^{r} / P_{i j} .
$$

If the two network flows (products) - for the resources $\left\{f_{i j}\right\}$ and for the risks $\left\{r_{i j}\right\}$ are limited from above by inequalities:

$$
f_{i j} \leq C_{i j}^{s}, \quad r_{i j} \leq C_{i j}^{r}, \quad(i, j) \in G,
$$

it follows from the previous four equations that the inequalities will also be observed for each $(i, j) \in G$

$$
f_{i j} \leq C_{i j}^{\prime}, \quad r_{i j} \leq C_{i j}^{\prime \prime} .
$$


For the realization of the transport circulation the following designations are necessary:

$I_{s}=\left\{i / x_{i} \in S\right\}$ - indices of the set of loading points;

$S=\cup_{i \in I_{s}} x_{i}$

$d_{i}$ - capacity (performance) by resources of a loading point of index $i \in I_{s}$;

$I_{d}=\left\{i / x_{i} \in T\right\}$ - indices in the set of unloading points;

$T=\underset{i \in I_{d}}{\cup} x_{i}$

$b_{i}-$ capacity (performance) by resources of a unloading point of index $i \in I_{t}$;

$I_{p}=\left\{i / x_{i} \in P\right\}$ - indices of the set of intermediate points;

$P=\underset{i \in I_{p}}{\cup} x_{i}$.

The requirement must be met for the transported product:

$$
\sum_{i \in I_{s}} d_{i}=\sum_{j \in I_{t}} b_{j}
$$

Let $l_{i j}$ be the number of time units for passing the distance from point $x_{i}$ to point $x_{j}$ which includes the time for loading/unloading at the same point $x_{j}$. Then $k_{i j}$ is a coefficient for transition from discrete to continuous transport, i.e.:

$$
k_{i j}=1 / l_{i j},(i, j) \in G .
$$

If it is assumed that the travel time on the individual sections of the network is the same for all sections, then for each $(i, j) \in G, l_{i j}=1$ and $k_{i j}=1$. It is advisable that the risk $r_{i j}$ is not less than the real arc risk $P_{i j} f_{i j}$ obtained through the resource flow $f_{i j}$, i.e. the requirement must be met:

$$
P_{i j} f_{i j} \leq r_{i j},(i, j) \in G,
$$

which means that

$$
P_{i j} f_{i j}-r_{i j} \leq 0,(i, j) \in G .
$$

The above requirement leads to the possibility to build a network risk flow, where it is possible to increase the risk of individual arcs, subject to the flow requirement. This makes it possible to build a two-product network flow of resources and risks with a total minimal cost.

The total costs for transporting the resource and for insuring the emerging risks are equal to:

$$
L_{s}=\sum_{(i, j) \in G} a_{i j}^{\prime} f_{i j} \text { and } L_{r}=\sum_{(i, j) \in G} a_{i j}^{\prime \prime} r_{i j}
$$




$$
L=L_{s}+L_{r}=\sum_{(i, j) \in G} a_{i j}^{\prime} f_{i j}+\sum_{(i, j) \in G} a_{i j}^{\prime \prime} r_{i j} \rightarrow \min \mid \max ,
$$

where $L_{s}$ are the costs of transporting resources, $L_{r}$ are the costs of risk insurance, and $L$ are the total costs $\left(L_{s}+L_{r}\right)$.

The quantity $L$ can be considered as a target function in the optimization problem of the total costs in the two-product flow.

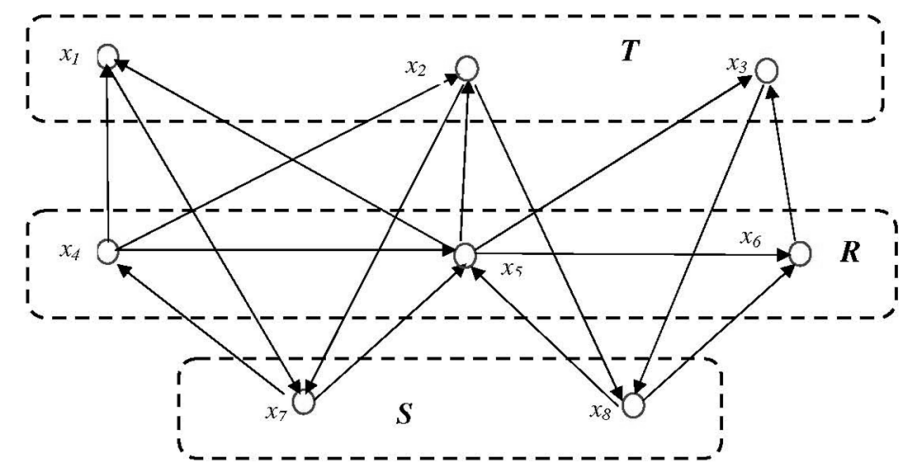

Fig. 1

In Figure 1 it is a schematic diagram of a transport network with two loading $S$, three unloading $T$ and three intermediate $P$ points. Flow can be circulated on the network. In that:

$$
X=S \cup P \cup T, S \cap P=\emptyset, S \cap T=\emptyset, T \cap P=\emptyset,
$$

where $\emptyset$ is an empty set symbol.

If the transport of the resource is carried out with discrete transport units and the travel times on different arcs of the set $U$ are different, then the transition to a continuous transport flow can be done by using a generalized (with profits and losses) two-product network flow with throughput and with coefficients $\left\{k_{i j} /(i, j) \in U\right\}$. It must comply with the flow requirements for both variables $\left\{f_{i j}\right\}$ and $\left\{r_{i j}\right\}$.

The introduced designations allow for the formalization of the two-product network flow of resources and risks, including their circulation. Determining the optimal/minimal circulation of resources and risks in this two-product flow is reduced to the following linear programming problem:

$$
L=\sum_{(i, j) \in G} a_{i j}^{\prime} f_{i j}+\sum_{(i, j) \in G} a_{i j}^{\prime \prime} r_{i j} \rightarrow \min
$$


with constraints for each $i \in I$ and $(i, j) \in G$;

$$
\begin{gathered}
\sum_{j \in \Gamma_{i}^{1}} f_{i j}-\sum_{j \in \Gamma_{i}^{-1}} k_{j i} f_{j i}=0, i \in I \\
\sum_{j \in \Gamma_{i}^{-1}} f_{j i}=d_{i}, i \in I_{s} \\
\sum_{j \in \Gamma_{i}^{-1}} f_{j i}=b_{i}, i \in I_{t} \\
P_{i j} f_{i j}-r_{i j} \leq 0, \quad(i, j) \in G ; \\
f_{i j} \leq C_{i j}^{s} ; \\
f_{i j} \geq 0, \quad(i, j) \in G \\
\sum_{j \in \Gamma_{i}^{1}} r_{i j}-\sum_{j \in \Gamma_{i}^{-1}} r_{j i}=0, i \in I \\
r_{i j} \geq 0, \quad(i, j) \in G .
\end{gathered}
$$

Observance of the requirement $\sum_{i \in I_{s}} d_{i}=\sum_{j \in I_{t}} b_{j}$ is necessary for the presence of circulation.

After solving the above problem for linear programming, consisting of 9 equations and inequalities, the optimal distribution of resources and risks $\left\{r_{i j}^{*} /(i, j) \in G\right\}$ along the $\operatorname{arcs} x_{i j} \in U$ will be obtained.

The value $\sum_{(i, j) \in G} f_{i j}^{*}=f^{*}$ indicates the maximal number of transport units that can ensure the transport of the produced resource $\sum_{i \in I_{s}} d_{i}$ from the loading points $S$ to the unloading points $T$ at a minimal total distance and with minimal transportation and risk insurance costs.

The optimal parameters obtained through the flow circulation can and should be considered as an optimal shift plan - it can be used to compile an optimal schedule for the movement of each individual dump truck. However, since there is a real stochasticity in the movement of vehicles, the obtained optimal schedules must be executed through the dispatching computer system for operational management of the transport units in an open, closed or mixed cycle. 
Problem 1: Equations and inequalities (4) and (11) to (18) define a twoproduct flow with resources and risks, which circulate on the network and lead to a minimal value of the two-product flow when transporting the resource:

$$
\sum_{i \in I_{s}} \sum_{j \in \Gamma_{i}^{-1}} f_{j i}=\sum_{i \in I_{t}} \sum_{j \in \Gamma_{i}^{-1}} f_{j i}=\sum_{i \in I_{s}} d_{i}
$$

from loading points $S$ through intermediate points $R$ to unloading points $R$.

Problem 2: The maximal possible value for circulation of the generalized two-product network flow of resources and risks can be determined by the following problem for linear programming:

$$
L=v_{\max }=\sum_{i \in I_{s}} \sum_{j \in \Gamma_{i}^{-1}} f_{j i} \rightarrow \max ,
$$

with constraints (15) to (19), (22) and (23)

$$
\begin{aligned}
& \sum_{i \in I_{s}} \sum_{j \in \Gamma_{i}^{-1}} f_{j i}-\sum_{i \in I_{t}} \sum_{j \in \Gamma_{i}^{-1}} f_{j i}=0 ; \\
& \sum_{i \in I_{s}} \sum_{j \in \Gamma_{i}^{-1}} r_{j i}-\sum_{i \in I_{t}} \sum_{j \in \Gamma_{i}^{-1}} f_{j i}=0 .
\end{aligned}
$$

The obtained value $v_{m}=\sum_{i \in I_{s}} \sum_{j \in \Gamma_{i}^{-1}} f_{j i}$ for the maximum possible circulation can be used in a number of dependences on the classical network flow, including the mincut-maxflow theorem of Ford and Fulkerson [1], namely:

$$
\begin{gathered}
v_{\max }=f\left(X_{0}, \bar{X}_{0}\right)=C\left(X_{0}, \bar{X}_{0}\right) ; \\
f\left(\bar{X}_{0}, X_{0}\right)=0 ; \\
\left(X_{0}, \bar{X}_{0}\right)=\left\{x_{i j} / x_{i} \in X_{0}, x_{j} \in \bar{X}_{0}\right\} ; \\
\left(\bar{X}_{0}, X_{0}\right)=\left\{x_{j i} / x_{i} \in X_{0}, x_{j} \in \bar{X}_{0}\right\},
\end{gathered}
$$

where $\left(X_{0}, \bar{X}_{0}\right)$ is the minimal cut.

Problem 3: If the maximal flow (21) in circulation is determined by Problem 2, then the maximal flow with minimal value can be calculated by the following linear optimization problem (11), (12), from (15) to (19) and in the following two additional equations:

$$
\sum_{i \in I_{s}} \sum_{j \in \Gamma_{i}^{-1}} f_{i j}=v_{\max }
$$

Engineering Sciences, LVII, 2020, No. 4 


$$
\sum_{i \in I_{t}} \sum_{j \in \Gamma_{i}^{-1}} f_{i j}=v_{\max }
$$

where $v_{\max }$ has a value obtained in (21) after solving Problem (2).

After solving Problem 3, the obtained value of $L$ in (11) is the minimal value of the maximal flow $v_{\max }$ from (21). The developed model makes it possible to minimize the number of circulating vehicles, ensuring the full transportation of the harvested agricultural products with minimal total costs for this transportation and to cover the emerging risks.

The following example illustrates how the proposed two-product network flow model with throughput and risks can be used effectively.

\section{NUMERICAL EXAMPLE}

Figure 2 shows a graph $G(X, U)$ with five vertices $\left\{x_{1}, x_{2}, x_{3}, x_{4}, x_{5}\right\}=X$ and eight $\operatorname{arcs} U=\left\{x_{1,5}, x_{2,5}, x_{3,1}, x_{3,2}, x_{4,1}, x_{4,2}, x_{5,3}, x_{5,4}\right\}$. On the graph there is one loading point $S=\left\{x_{5}\right\}$ and two unloading points $T=\left\{x_{1}, x_{2}\right\}$ for which the requirements (10) are observed.

Table 1 shows the coefficients needed to determine the optimal circulation of the two-product flow.

Problem 1: The optimal two-product network flow with circulation and risks can be determined by the dependences from (11) to (19), which for the

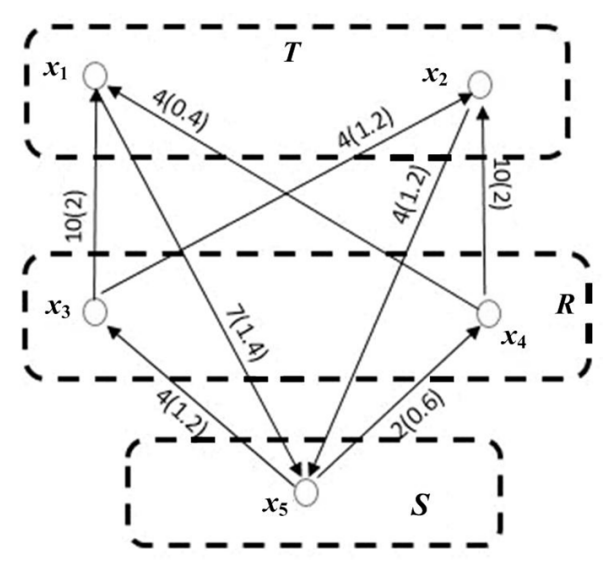

Fig. 2 
Table 1. Source data

\begin{tabular}{|c|c|c|c|c|c|c|c|c|c|c|c|c|c|c|c|c|}
\hline \multirow{2}{*}{\begin{tabular}{|c|} 
Para- \\
me- \\
ters \\
\end{tabular}} & \multicolumn{8}{|c|}{ Arcs - resource } & \multicolumn{8}{|c|}{ Arcs - risks } \\
\hline & $(1,5)$ & $(2,5)$ & $(3,1)$ & $(3,2)$ & $(4,1)$ & $(4,2)$ & $(5,3)$ & $(5,4)$ & $(1,5)$ & $(2,5)$ & $(3,1)$ & $(3,2)$ & $(4,1)$ & $(4,2)$ & $(5,3)$ & $(5,4)$ \\
\hline$c_{i j}^{\prime}$ & 7 & 4 & 10 & 8 & 4 & 10 & 8 & 4 & - & - & - & - & - & - & - & - \\
\hline$c_{i j}^{\prime \prime}$ & - & - & - & - & - & - & - & - & 1.8 & 1.2 & 2.2 & 1.2 & 1 & 2.2 & 1.2 & 0.6 \\
\hline$c_{i j}^{s}$ & 7 & 4 & 10 & 4 & 4 & 10 & 4 & 2 & - & - & - & - & - & - & - & - \\
\hline$c_{i j}^{r}$ & - & - & - & - & - & - & - & - & 1.4 & 1.2 & 2.0 & 1.2 & 0.4 & 2.0 & 1.2 & 0.6 \\
\hline$a_{i j}^{\prime}$ & 16 & 12 & 10 & 9 & 12 & 10 & 9 & 14 & - & - & - & - & - & - & - & - \\
\hline$a_{i j}^{\prime \prime}$ & - & - & - & - & - & - & - & - & 8 & 8 & 12 & 8 & 8 & 12 & 8 & 6 \\
\hline \multirow[t]{2}{*}{$p_{i j}$} & 0.2 & 0.3 & 0.2 & 0.3 & 0.1 & 0.2 & 0.3 & 0.3 & 0.2 & 0.3 & 0.2 & 0.3 & 0.1 & 0.2 & 0.3 & 0.3 \\
\hline & \multicolumn{8}{|c|}{ Vertices - resource } & \multicolumn{8}{|c|}{ Vertices - risks } \\
\hline$x_{i}$ & $x_{1}$ & $x_{2}$ & $x_{3}$ & $x_{4}$ & $x_{5}$ & - & - & - & $x_{1}$ & $x_{2}$ & $x_{3}$ & $x_{4}$ & $x_{5}$ & - & - & - \\
\hline$d_{i}\left(b_{i}\right)$ & 0 & 0 & 0 & 0 & 5 & - & - & - & 0 & 0 & 0 & 0 & 1.7 & - & - & - \\
\hline
\end{tabular}

graph of Fig. 2 and the data in Table 1 have the following form:

$$
\begin{aligned}
L= & 16 f_{15}+12 f_{25}+10 f_{31}+9 f_{32}+12 f_{41}+10 f_{42}+9 f_{53}+14 f_{54}+8 r_{15} \\
& +8 r_{25}+12 r_{31}+8 r_{32}+8 r_{41}+12 r_{42}+8 r_{53}+6 r_{54} \rightarrow \min ;
\end{aligned}
$$

subject to constraints and equations
1. $f_{15}-f_{31}-f_{41}=0$;
2. $f_{25}-f_{32}-f_{42}=0$;
3. $f_{31}+f_{32}-f_{53}=0$;
4. $f_{41}+f_{42}-f_{54}=0$;
5. $f_{53}+f_{54}-f_{15}-f_{25}=0$;
6. $f_{15}+f_{25}=5$;
7. $f_{31}+f_{41}+f_{32}+f_{42}=5$;
8. $0.2 f_{15}-r_{15} \leq 0$;
9. $0.1 f_{25}-r_{25} \leq 0$;
10. $0.2 f_{31}-r_{31} \leq 0$;
11. $0.3 f_{32}-r_{32} \leq 0$;
12. $0.1 f_{41}-r_{41} \leq 0$;
13. $0.2 f_{42}-r_{42} \leq 0$;
14. $0.3 f_{53}-r_{53} \leq 0$;
15. $0.3 f_{54}-r_{54} \leq 0$;
16. $f_{15} \leq 7$;
17. $f_{25} \leq 4$;
18. $f_{31} \leq 10$; 
19. $f_{32} \leq 4$

20. $f_{41} \leq 4$

21. $f_{42} \leq 10$

22. $f_{53} \leq 4$

23. $f_{54} \leq 2$;

24. $r_{15}-r_{31}-r_{41}=0$;

25. $r_{25}-r_{32}-r_{42}=0$;

26. $r_{31}+r_{32}-r_{53}=0$;

27. $r_{41}+r_{42}-r_{54}=0$;

28. $r_{53}+r_{54}-r_{15}-r_{25}=0$;

29. $f_{i j} \geq 0 ;(i, j) \in G, r_{i j} \geq 0 ;(i, j) \in G$.

The results obtained from solving the optimization problem described above are given in Table 2. It shows the obtained values of resources and risks along the individual arcs of the network. The last two rows of the table show the differences between the throughputs and the corresponding arc flow functions of the two products. Where these differences are zero, there is a flow saturation for the respective product - resource or risk.

Table 2. Results for Problem 1

\begin{tabular}{|c|c|c|c|c|c|c|c|c|c|c|c|c|c|c|c|c|}
\hline \multirow{2}{*}{$\begin{array}{c}\text { Para- } \\
\text { me- } \\
\text { ters } \\
\end{array}$} & \multicolumn{8}{|c|}{ Arcs - resource } & \multicolumn{8}{|c|}{ Arcs - risks } \\
\hline & $(1,5)$ & $(2,5$ & $(3,1)$ & $(3,2)$ & $(4,1$ & $(4,2$ & $(5$, & $(5,4$ & $(1,5)$ & $(2,5)$ & $(3,1)$ & $(3,2)$ & $(4,1)$ & $(4,2$ & $(5,3)$ & $(5,4)$ \\
\hline$f_{i j}$ & 1 & 4 & 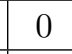 & 4 & 1 & 0 & 4 & 4 & - & - & - & - & - & - & - & - \\
\hline$r_{i j}$ & - & - & - & - & - & - & - & 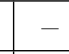 & 0.3 & 1.2 & 0 & 1.2 & 0.3 & 0 & 1.2 & 0.3 \\
\hline$p_{i j}$ & 0.2 & 0.3 & 0.2 & 0.3 & 0.1 & 0.2 & 0. & 0.3 & 0.2 & 0.3 & 0.2 & 0.3 & 0.1 & 0.2 & 0.3 & 0.3 \\
\hline$c_{i j}^{s}-f_{i j}$ & $66_{1}$ & 0 & 10 & 0 & 3 & 10 & 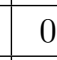 & 1 & - & - & - & - & - & - & - & - \\
\hline$c_{i j}^{r}-r_{i j}$ & - & - & - & - & - & - & - & - & 0.9 & 0 & 2.2 & 0 & 0.7 & 2 & 0 & 0.3 \\
\hline
\end{tabular}

From the last two rows of Table 2 it follows that when transporting the resources the sections $\left\{x_{25}, x_{32}, x_{53}\right\}$ are saturated. The same sections are saturated with the risks.

Figure 3 shows the graph of Fig. 2 on the arcs on which the obtained values of the resource flow are plotted, and in brackets - the flow of risks.

The saturated arcs of the graph of Fig. 3 are marked with bold lines. They form a loop on the same graph. A flow with a value of 4 circulates on the cycle. There is also a second cycle $-\left\{x_{41}, x_{15}, x_{54}\right\}$ on which a resource flow with a value of 1 and a risk flow with a value of 0.3 circulate. This second cycle has unsaturated arcs. 


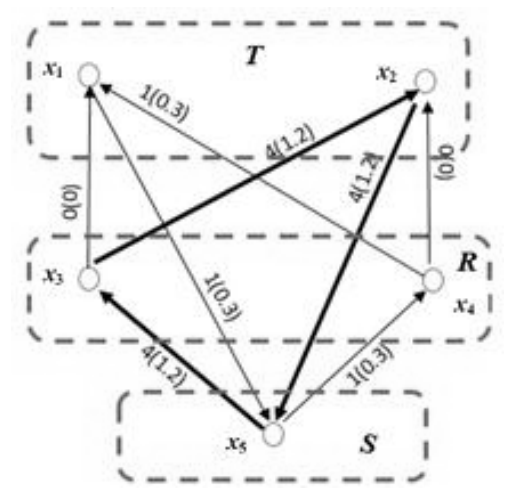

Fig. 3

Problem 2: To determine the maximum possible flow of resources and risks, almost the same dependencies will be used as in Problem 1, but with some changes. In this case, the objective function has the following form:

$$
L=v_{\max }=f_{15}+f_{25} \rightarrow \max .
$$

In constraints (1) to (44), equations (6) and (7) must be replaced by a single equation:

$$
f_{15}+f_{25}-f_{31}-f_{41}-f_{32}-f_{42}=0 .
$$

The results of solving the thus formulated Problem 2 with data from Table 1 are given in the following Table 3 .

In Figure 4 saturated arcs are bold, non-zero unsaturated arcs are dotted, and the rest are zero flow. The maximal flow obtained is equal to 6 .

Problem 3: The maximal value of the flow of resources obtained in the previous Problem 2 will be used to obtain the maximal flow with a minimal

\begin{tabular}{|c|c|c|c|c|c|c|c|c|c|c|c|c|c|c|c|c|c|}
\hline \multirow{2}{*}{$\begin{array}{c}\text { Para- } \\
\text { me- } \\
\text { ters }\end{array}$} & \multicolumn{9}{|c|}{ Arcs - resource } & \multicolumn{8}{|c|}{ Arcs - risks } \\
\hline & $(1,5)$ & $(2,5)$ & $(3,1$ & $(3,2$ & $(t, 1$ & $(4$, & & & $(5,4)$ & $(1,5)$ & $(2,5)$ & $(3,1)$ & $(3,2)$ & $(4,1)$ & $(4,2)$ & $(5,3)$ & $(5,4)$ \\
\hline$f_{i j}$ & 6 & 0 & 4 & 0 & 2 & 0 & & 4 & 2 & - & - & - & - & - & - & - & - \\
\hline$r_{i j}$ & - & - & 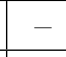 & - & - & - & & - & - & 1.4 & 0.4 & 1.2 & 0 & 0.2 & 0.4 & 1.2 & 0.6 \\
\hline$p_{i j}$ & 0.2 & 0.3 & 0.2 & 0.3 & 0.1 & 0. & & 0.3 & 0.3 & 0.2 & 0.3 & 0.2 & 0.3 & 0.1 & 0.2 & 0.3 & 0.3 \\
\hline$c_{i j}^{s}-f_{i j}$ & 1 & 4 & 6 & 4 & 2 & 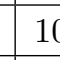 & & 0 & 0 & - & - & - & - & - & - & - & - \\
\hline$c_{i j}^{r}-r_{i j}$ & - & - & - & - & - & - & & - & - & 0 & 0.8 & 0.8 & 1.2 & 0.2 & 1.6 & 0 & 0 \\
\hline
\end{tabular}

Table 3. Results for Problem 2 


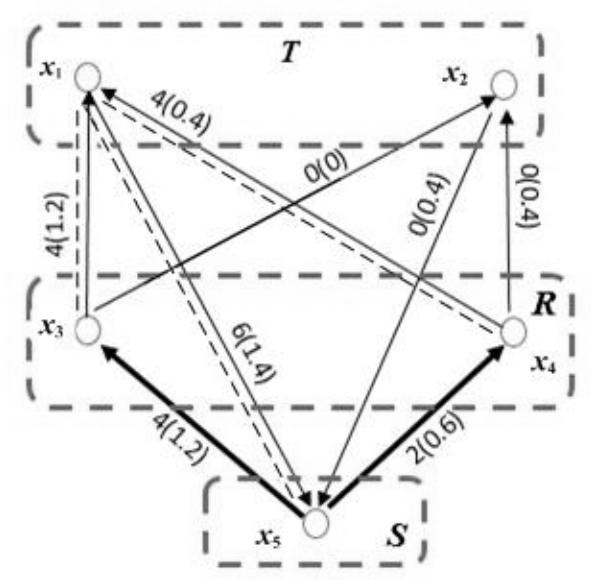

Fig. 4

value. In this case, the objective function has the same coefficients and variables as in Problem 1, but in the constraints - the two equations (6) and (7) have right parts equal to $v_{\max }$, namely:

$$
\begin{gathered}
f_{15}+f_{25}=v_{\max } ; \\
f_{31}+f_{41}+f_{32}+f_{42}=v_{\max } .
\end{gathered}
$$

After solving the optimization problem, the maximal generalized two-product flow with circulation, risks and a minimal value is determined.

The obtained solution coincides with the solution of Problem 2. The data for it are marked in Table 3 .

\section{CONCLUSION}

1. For optimal planning and management of the freight transport activity during the harvesting of the agricultural production it is proposed to use the circulation in a generalized two-product network flow of resources and risks with throughput, which allows to optimize the total transport and insurance costs of emerging risks. The model takes into account the capacity and the unit value for transportation of each of the sections of the network.

2. Risk is defined in the same way as in man-made catastrophic events as a product of two measures - the volume of transported agricultural production on a given section of the network and the probability of an adverse event on the same section. 
3. A method has been proposed for determining the maximal number of means of transport for the transport of harvested agricultural products. The method determines the optimal route of each transport unit with a total minimal cost for the entire transport activity, including insurance against emerging risks.

4. It is proposed that the general transport process be controlled by an appropriate computer system and, in the event of various reasons for significant disturbances in the traffic schedule, that it be recalculated according to the proposed method.

\section{REFERENCES}

[1] Tasso Adamopoulos, Transportation Costs, Agricultural Productivity, and Cross-Country Income Differences, International Economic Review (May 2011) 52 (2) 489-521, https://doi.org/10.1111/j.1468-2354.2011.00636.x

[2] V. Sgurev and St. Drangajov, Intelligent Control of Flows with Risks on a Network, in: Proceedings of the $7^{\text {th }}$ IEEE International Conference Intelligent Systems - IS'14, September 24-26 2014, Warsaw, Poland, ISSN 2194-5357, ISSN 2194-5365 (electronic), ISBN 978-3-319-11309-8, ISBN 978-3-319-11310-4 (eBook), DOI: 10.1007/978-3-319-11310-4, Volume 2: Tools, Architectures, Systems, Applications, Springer International Publishing, Switzerland (Eds P. Angelov et al.), Advances in Intelligent Systems and Computing (2014) 323 27-35, R. YAGER, Multi-Criteria Decision Making with Uncertainty, IEEE Transactions on Fuzzy Systems (April 2018) 26 (2) 1023-1031.

[3] V. Sgurev and S. Drangajov, Network Flows with Risks, BAS Publishing House, Sofia (2019), ISBN 978-954-322-990-1 (in Bulgarian).

[4] P. Jensen and J. Barnes, Networks Flow Programming, John Wiley and Sons Inc. New York (1980).

[5] N. Christofides, Graph Theory: an Algorithmic Approach, Academic Press, London (1986).

[6] L. Ford And D. Fulkerson, Maximal Flow through a Network, Canadian Journal of Mathematics (1956) 8 399-404.

Received December 21, 2020

Engineering Sciences, LVII, 2020, No. 4 